\title{
The Causes of Social Anxiety Disorder in the Different Developmental Stages
}

\author{
Xinyi Cheng ${ }^{1, \dagger}$, Yuwen Yang ${ }^{2, *, \dagger}$ \\ ${ }^{1}$ School of Education, Hebei Normal University of Science and Technology, Qinhuangdao 066000, China \\ ${ }^{2}$ School of Education, Suzhou University of Science and Technology, Suzhou 215000, China \\ *Corresponding author. Email: guanghua.ren@gecacdemy.cn \\ These authors contributed equally.
}

\begin{abstract}
Socializing is an essential and fundamental aspect of our daily routine. Previous studies have indicated that certain people were suffering from social anxiety, which continues to have significant and serious impacts on people's lives. The health and social consequences of Social Anxiety Disorder have widely attracted public attention and also caused sensation and discussion in the medical and psychological circles. The research analyzed and contrasted prior research from the standpoint of development which influences people most and provides a timeline. This paper summarizes the causes of social anxiety in different developmental stages. The conclusion is that it is important to pay attention to the internal and external factors in the key stages of individual life and predict Social Anxiety Disorder from the roots. In addition, some flaws in previous research are discovered.
\end{abstract}

Keywords: Social Anxiety Disorder, Social Phobia, developmental stage, genetic influences, self-evaluation

\section{INTRODUCTION}

Humans are social animals, and meaningful communication with others is the foundation of human social life. Baumeister, a psychologist, pointed out that belonging is the most important, basic, and widespread social motivation of mankind [1]. Human-to-human interaction, whether in science, philosophy, or even theology, is a proposition worth exploring. Similarly, socialization has received a lot of attention in psychology.

Social anxiety disorder (SAD) was first proposed by British neurologists Mark and Gelder. The two psychologists found that people with social anxiety tend to be alone and very afraid of criticism [2]. For example, when patients are in public, they will delay examination by others and fear making a fool of themself. And this emotion can also lead to the individual avoiding these occasions or extreme tension and discomfort, accompanied by an accelerated heartbeat, blushing cheeks, dry mouth, trembling, difficulty swallowing, muscle tension, constant and persistent anxiety, and other physiological symptoms [3][4]. According to some researchers, there are two types of social anxiety: Generalized Anxiety Disorder and Nongeneralized Social Anxiety Disorder. Then a new notion was proposed: Discrete Social Anxiety Disorder and is also known as specific social anxiety, which refers to fear solely in specific social settings, such as public speaking phobias [5].

One of the topics we discuss daily is shyness, which is strongly linked to social anxiety. Shyness is a typical subjective feeling, and everyone feels shy in their daily life to varying degrees [6]. Shyness was first defined by Lewinsky in 1941 as a state of severe restraint, a sense of inferiority, disregard, and oversensitivity to one's sentiments and emotions. Frequent physiological symptoms accompany it: blushing, sweating, shivering, inability to talk fluently, and so on. Around each other, social anxiety and shyness are a deeper, more tenacious, and intense fear of a certain circumstance, as well as a tenacious notion that embarrassment and humiliation occur in social or public contexts [7].

After statistics and investigation, the incidence of Social Anxiety Disorders highly relates to adolescents and early adulthood, and it is less common after the age of 25 [7][8]. Patients with social anxiety disorders have a lifetime risk of 3 percent to 13 percent, with a 1:1.5 to 2.0 male-to-female incidence ratio. Furthermore, the symptoms of social anxiety disorder can appear as early as childhood or even in infancy [9]. The initial age is 11 to 15 years old (average 14 years old) and more than $80 \%$ 
of patients become ill before the age of 25 , but the earliest age of onset is also mentioned as 5 to 9 years old [3]. People with social anxiety disorders are significantly more likely than those without social anxiety disorders to develop other mental disorders throughout their lives such as pure terror, developing square terror, alcohol abuse, severe depression, drug abuse, and obsessivecompulsive disorder [10].

People's social patterns in modern times have changed subtly and fundamentally as a result of the development and renewal of technology and the internet. The difference in space will influence individuals mentally and psychologically. Many academics are currently researching the social differences between the internet and reality. From a physical standpoint. Because of its virtuality, anonymity, and other attributes, cyberspace can lead to the decrease of the importance of individual physical signs (such as appearance), the decrease of the importance of nonverbal communication, and the improvement of self-disclosure and initiative. From a behavioral perspective, social networking is outof-sync. According to Walther's hyper-personal model, because of this feature, individuals have plenty of time to think and edit self-presenting information, reducing social anxiety caused by language and expression that they are not good at in real space [11]. The term "social phobia," a popular cyber world in China, was coined and began to appear more regularly in the public eyes, with an increasing number of people using it to describe their anxiety and anguish as a result of socializing, whether in person or online.

Recently, Educational circles and the medical community in China have also begun to pay attention to the social interaction and social anxiety of young people, but academia rarely approaches social anxiety disorders from the developmental stage of life. Physiological mechanisms and drugs for social anxiety disorders are studied in medicine, and psychological models are also proposed to explain social anxiety phenomena and causes. However, most studies have abandoned longitudinal developmental clues in favor of a horizontal view of the entire life process, and these studies and findings come from several psychological research traditions that rarely communicate with one another. This study aimed to review the causes of Social Anxiety Disorder from the perspective of developmental stages.

\section{THE CAUSES OF SOCIAL ANXIETY DISORDER IN THE DIFFERENT DEVELOPMENTAL STAGES}

There is no single element that causes social anxiety; rather, several factors interact to generate it. According to Developmental Psychopathology and Develop Cascaded Model, development is influenced by a variety of elements at various levels of analysis, all of which combine to produce the development process. As a result, many alternative paths can lead to the same pathological outcomes, the study should also consider the same result principle as well as the principle of diverse outcomes [7].

Psychologist Erik H Erikson proposed the eight stages of life development theory, which divided human development into the infant, early childhood, pre-school, school age, and other four stages of life, assigning tasks and conflicts to each stage of development and explaining that if the conflict persists or is not resolved perfectly, it will harm the individual. The purpose of this paper is to better understand the causes of SAD by using the developmental stage of life as a clue, highlighting the characteristics of observing and paying attention to the social development and critical period of the individual, and integrating the most prominent influence factors on the stage.

\subsection{Infants and Toddlers}

During this time, their genetic influences in the early development of persons have a significant impact, while the early growth of individuals experienced in the living environment and their parents' parenting style has a significant influence on the growth of individuals.

According to studies, social anxiety problems are moderately hereditary [12]. A study of 2,000 twins over eight years found that the hereditary rate of social anxiety had risen to $51 \%$ [13]. The expression of genes encoding oxytocin and Gastrin-Releasing Peptide (GRP) receptors, for example, may have an impact on social anxiety [14].

Kagan established the Behavioral Inhibition (BI) to the unfamiliar temperament type theory in 1988 at Harvard University, which describes a tendency toward social anxiety that develops in childhood [15]. It manifests itself as extreme behavioral inhibition in the presence of strangers or unfamiliar environments, and this type of temperament also has some stability [16] and a certain stage [7][12][15].

Furthermore, electroencephalogram scans of sixmonth-old babies with this temperament type differ from those of other infants, with comparatively high activation of the right frontal lobe and relatively low activation of the left frontal lobe [12].

Until the age of 6, parents and families have an important role as social agents, who have the mission to improve children's ability and socialization of personality. At this stage, children must learn to make their own decisions and explore the type of person they will become in the future; social anxiety is likely to start in childhood, and children will exhibit general inhibitions of their behaviors in unfamiliar situations [3]. Meanwhile, animal and human studies have demonstrated that environmental influences play an essential role in the plasticity of young children's social development [16]. To put it another way, the way parents raise their children 
and the family atmosphere in which they grow and develop is crucial. According to research, most parents in SAD patients are rejected or overprotective of parenting conduct and misleadingly educate their children. They are indifferent and more concerned with other people's opinions than typical parents, and they are also prone to shame for their children's bad performance [7].

Furthermore, early stressful experiences such as losing parents, seeing domestic violence, being sexually assaulted, and being bullied can have a long-term impact on their development [17]. According to studies, adults who have been through these experiences have much fewer hippocampus, front buckles, and tail cores than the general population [18]. Additionally, an examination of the relationship between parental psychiatry and parenting approaches reveals that the existence of mental illness in parents, as well as their parenting style, increases the risk of SAD in their children [19].

\subsection{Pre-school and School-age}

It is at the key stage of children's development that the individuals' self-consciousness and genderconsciousness initially awaken. Children began to develop their abilities to cooperate with others. Teachers and peers have become the most significant social agents. During this period, social interaction becomes more and more important for children, and the influence of peer groups has gradually begun to emerge as well. Additionally, while individuals are only involved in a group, the impact of self-evaluation has intensified, and their social attention patterns have begun to distinguish.

In middle childhood, there is a significant positive association between the occurrence of social anxiety and relational aggressiveness, as well as physical aggression in peers. And the gender differences are gradually reflected as well, especially in physical aggression. But it can only predict the social anxiety of girls, not boys [20].

The early experience of Clark's SAD information processing model pointed out that children who suffered from SAD will put forward a series of negative assumptions pointing to their own when they are in adolescence. There is a significant positive correlation between the occurrence of social anxiety and relational aggression together with physical aggression in peers infringement in their middle childhood. At the same time, they also use negative external evaluations to confirm their assumptions which made them fall into a vicious circle [21]. On this basis, Clark and Wells also proposed two modes of attention: Observer Perspective and Field Perspective. Studies have found that SAD patients tend to use Observer Perspective in social situations, which is the perspective of simulating the eyes of others. And in non-social situations, they take Field Perspective of attention, which is from their own [22]. This observation mode will shift attention from others and the environment to the reflection and control of themselves. The excessive attention of their own in addition to increasing tension and also reducing their efforts to adapt which reduce their attention to the task itself, and ultimately lead to damage to the implementation of the task [23].

In the hypothesis of behavior and cognitive theory, the phobic reaction is acquired after reinforcement. So according to the cognitive model, it is proposed that SAD patients have special cognitive schema or prejudice, which events are regarded as dangerous and beyond personal coping ability. Consider social settings as potential threats or dangers that will trigger a cascade of emotional, physiological, and behavioral responses [3]. And studies have confirmed that those who suffered from social anxiety have more negative assessments of social situations than those without considering social settings as potential threats or dangers that will trigger a cascade of emotional, physiological, and behavioral responses. social anxiety [24].

\subsection{Adolescence}

Individuals begin to examine their beliefs and professional aspirations when they enter adolescence, and subsequently establish personal self-identity. Social status, self-esteem level, and social style will play very important roles in this development process and even have a great influence on their mental health and defense mechanism.

Studies have shown that the score of individual social anxiety and the social avoidance with distress dimension have significant main effects during this period. In specific empirical studies, the overall anxiety level of the rejected group is higher than that of the popular group [25]. The degree of separation between implicit selfesteem and explicit self-esteem also has an important impact on social anxiety. Explicit self-esteem has a buffer effect on social anxiety. For example, the explicit self-esteem of junior middle school freshmen has an obvious impact on reducing social anxiety. However, although implicit self-esteem itself does not directly lead to social anxiety, this bad result may occur when implicit self-esteem is higher than explicit self-esteem [26]. Nowadays people's social space has taken place qualitative changes. Under the impact of the development of the Internet, studies have shown that almost $26 \%$ of the students have participated in social networking. Juvenile adults have become the primary population of China's Internet users. For individuals in adolescence, they are more likely to reduce social interaction in reality because of the advantages of social networking, and the differences between social networking and social interaction in real life will lead to social isolation and social anxiety [27].

Social anxiety has its characteristics in the indirect predictive effect on mental health through the coping 
style [28]. In a study on the characteristics of social anxiety of junior high school students, it is found that the adult attachment in the SAD group is dominated by types of insecurity characterized by pre-occupational and fearful insecurity, while the rejection type is less [29]. Other studies claimed that the mean scores of intermediate and immature defense mechanism factors and masking factors in the SAD group were higher than those in the normal control group, and the mean scores of mature defense mechanism factors were lower than those in the normal control group. Among them, defense mechanisms such as disengagement, suppression, avoidance, isolation, projection, subconscious presentation, withdrawal, and somatization were widely used [30].

\subsection{Emerging Adulthood}

In the year 2000, Arnett offered the concept of Emerging Adulthood for the first time. This phase usually occurs in cultures that allow young people to play their independent role for a long time (e.g., cultures in western developed countries) and it is concentrated mainly in the 18-25 years of age. In emerging adulthood, the formation of individual identity involves trying various possibilities of life and moving towards making lasting decisions. This process begins in adolescence but mainly occurs in adulthood. In American popular media, the term 'quarterlife crisis' was coined and used to describe the difficulties they experienced in trying to find a place in the adult world [31]. Some people also experienced serious mental health problems, such as severe depression and drug use disorders [32][33]. We divide the factors affecting individual social anxiety into self and social support here according to the negative side of emerging adulthood.

Self-evaluation and social skills provide a basic guarantee and premise for individual social interaction from inside to outside. The worse one's self-esteem and social abilities are, the higher one's social anxiety will be [7][34]. College students with obstacles in social skills will be excluded from social interaction in reality. They show high rejection sensitivity and have a negative aversion to social behavior in reality. At the same time, the fear of re-exclusion and avoidance of communication finally results in tension negative emotions. According to the social compensation model, they usually look for comfort in cyberspace that is divorced from reality to alleviate anxiety and meet their inner social needs. Due to the anonymity of the network environment, they can have a higher level of self-disclosure, which compensates for their poor social skills in the real life. However, at the same time, there are also some students who once again suffer from the negative emotional experience of social networking, which further enhances their social anxiety and rejection [35].

Social support system provides emotional security and support for individuals from the outside to the inside, thereby reducing social avoidance behavior. After entering the university, the development trend of social support individuals received from vertical and family support to horizontal and external support. Most social anxiety is due to the failure to deal with this change. They still rely on family support and do not realize the importance of mutual peer support. College students with social anxiety fail to build their own good social support system. On the one hand, they are eager for communication; but on the other hand, they are depressed and lonely. This psychological contradiction is very common [36]. Another possible explanation is that the differences in mental health functions have become more widespread. But some other individuals in emerging adulthood find themselves lost in direction, which may fall into serious mental health problems [37].

Furthermore, individuals begin to formally accept social norms at this stage (e.g., women should be implicitly introverted), which is the primary reason why women are more prone to suffer from SAD than men [38].

\section{CONCLUSION}

By summarizing the above researches, we took the development process of life as the time clue, longitudinally combs the different factors of social anxiety in different stages of life. A deeper study of developmental characteristics is required to prevent and reduce social anxiety disorder from the root.

In most empirical studies, social anxiety is always regarded as a research problem in psychiatry, in which studies on physiology and neuroticism are very rich, while studies on psychology are relatively few, especially focusing on the characteristics of various age stages. Therefore, future researchers can study individuals more from the theoretical perspective of psychology.

In the study of the impact of parents' mental illness on future generations, we have known that most mental illnesses are genetically inherited. And the parenting style of parents with mental illnesses to future generations may also affect the germination of children's social anxiety. It should be different from simply focusing on different dimensions of parenting styles, and the influence of abnormal behavior patterns caused by psychiatric factors on children is discussed. There is still much space for exploration in this area.

The mechanism of the impact of changes in the social environment of new online social platforms on social anxiety deserves further exploration. It seems that social anxiety disorder has not been taken very seriously in China. There are few studies on its causes, prevention, and treatment, especially in differences between urban and rural areas. It is necessary to pay more attention to the causes of anxiety tendency in the process of individual development under different environmental backgrounds. 


\section{REFERENCES}

[1] Baumeister Roy F.,Leary Mark R.. The need to belong: Desire for interpersonal attachments as a fundamental human motivation.[J]. Psychological Bulletin,1995,117

[2] Watson D, Friend R. Measurement of socialevaluative anxiety.[J]. Journal of Consulting \& Clinical Psychology, 1969, 33(4):448.

[3] JI Jiang-lin. Understanding Social Anxiety Disorder[J]. Shanghai Journal of Preventive Medicine,2002(02):93-95.

[4] GUO Jing. Study on the Causes and Intervention Methods of Social Anxiety[J]. Literatures (theoretical edition),2012(11):411-412.

[5] Weinstock L S. Gender differences in the presentation and management of social anxiety disorder[J]. J Clin Psychiatry, 1999, 60 Suppl 9(60 Suppl 9):9-13.

[6] LUO Fang, JIANG Li-ming, TIAN Xue-tao, XIAO Meng-ge, MA Yan-sheng, ZHANG Sheng. Shyness prediction and language style model construction of elementary school students[J]. Acta Psychologica Sinica,2021,53(02):155-169.

[7] Thomas H Ollendick,Dina R Hirshfeld-Becker. The developmental psychopathology of social anxiety disorder[J]. Biological Psychiatry,2002,51(1).

[8] GUO Xiao-wei. Study on Cause of Social Anxiety[J].Psychological Exploration,2000(01):5558 .

[9] Kagan J, Reznick J , Snidman N . Biological bases of childhood shyness[J]. Science, 1988, 240(4849):167-171.

[10] J, R, T, et al. The epidemiology of social phobia: findings from the Duke Epidemiological Catchment Area Study[J]. Psychological Medicine, 1993.

[11] JOSEPH, B, WALTHER. Computer-Mediated Communication: Impersonal, Interpersonal, and Hyperpersonal Interaction[J]. Communication Research, 1996, 23(1):3-43.

[12] Goldsmith $\mathrm{H}$ H, Lemery $\mathrm{K}$ S. Linking temperamental fearfulness and anxiety symptoms: a behavior-genetic perspective[J]. Biological Psychiatry, 2000, 48(12):1199-1209.

[13] Kendler K S, Karkowski L M, Prescott C A. Fears and phobias: reliability and heritability.[J]. Psychological Medicine, 1999, 29(3):539-53.

[14] Domes G, Heinrichs M, Gl?Scher J, et al. Oxytocin attenuates amygdala responses to emotional faces regardless of valence.[J]. Biological Psychiatry, 2007, 62(10):1187-1190.
[15] Kagan J,Snidman N,Zentner M,Peterson E. Infant temperament and anxious symptoms in school age children.[J]. Development and psychopathology,1999,11(2).

[16] De Gnan K A, Fox N A. Behavioral inhibition and anxiety disorders: Multiple levels of a resilience process[J]. Dev Psychopathol, 2007, 19(3):729-746.

[17] HE Quan-min, PAN Run-de, MENG Xian-zhang. Relationship of Social Anxiety Disorder and Child Abuse and Trauma[J]. Chinese Journal of Clinical Psychology,2008(01):40-42.

[18] Cohen R A, Grieve S, Hoth K F, et al. Early Life Stress and Morphometry of the Adult Anterior Cingulate Cortex and Caudate Nuclei[J]. Biological Psychiatry, 2006, 59(10):975-982.

[19] Knappe Susanne,Lieb Roselind,Beesdo Katja,Fehm Lydia,Low Nancy Chooi Ping,Gloster Andrew T,Wittchen Hans-Ulrich. The role of parental psychopathology and family environment for social phobia in the first three decades of life.[J]. Depression and anxiety,2009,26(4).

[20] ZHANG Wen-xin, CHEN Liang, JI Lin-qin, ZHANG Ling-ling, CHEN Guang-hui, WANG Shu-qiong. Physical and Relational Victimization, and Children's Emotional Adjustment in Middle Childhood $[\mathrm{J}]$. Acta $\quad$ Psychologica Sinica,2009,41(05):433-443.

[21] Clark D M, F Mcmanus. Information processing in social phobia[J]. Biological Psychiatry, 2002, 51(1):92-100

[22] Wells A, Papageorgiou C. The observer perspective: Biased imagery in social phobia, agoraphobia, and blood/injury phobia[J]. Behaviour Research and Therapy, 1999, 37(7):653-658.

[23] Mansell W, Clark D M, Ehlers A. Internal versus external attention in social anxiety: an investigation using a novel paradigm[J]. Behaviour Research \& Therapy, 2003, 41(5):555-572.

[24] Miers A C, Bl?Te A W , B?Gels S M , et al. Interpretation bias and social anxiety in adolescents[J]. Journal of Anxiety Disorders, 2008, 22(8):1462-1471.

[25] WU Wei-li, ZHANG Wei. Comparison of AAS between $\mathrm{SAD}$ group and $\mathrm{NC}$ group[J]. Journal of Sichuan University (Medical Sciences) ,2005(02):271-273.

[26] SUN Pei-zhen, JIANG Hong-yan, ZHAO Hui. Relationship among Implicit Self-esteem, Explicit Self-esteem and Social Anxiety of Junior Middle 
School Students[J]. China Journal of Health Psychology,2008(01):116-118.

[27] Turkle, S. (2012). Alone together: Why we expect more from technology and less from each other. New York: Basic books.

[28] CHEN Xia, MA Shu-juan, DAI Mei-lin. A Research on Relationship among Social Anxiety,Coping Style and Mental Health of Countryside Middle School Students $[\mathrm{J}]$. China Journal of Health Psychology,2007(02):138-140.

[29] XIN Zi-qiang, CHI Li-ping, LIU Bing-yuan. The Differences of Social Anxiety of Adolescents with Different Sociometric Status[J]. Chinese Mental Health Journal,2004(04):231-232+221.

[30] LI Bo, QIAN Ming-yi, ZHONG Jie. Undergraduates' Social Anxiety: a Shame Proneness Modle. [J]. Chinese Mental Health Journal,2005(05):14-16.

[31] Robbins A, Wilner A, Tarcher J P . Quarterlife crisis: the unique challenges of life in your twenties.

[32] Schulenberg J E , Zarrett N R . Mental Health During Emerging Adulthood: Continuity and Discontinuity in Courses, Causes, and Functions.[M]. 2006.

[33] Tanner J L, Reinherz H Z, Beardslee W R, et al. Change in prevalence of psychiatric disorders from ages 21 to 30 in a community sample.[J]. Journal of Nervous \& Mental Disease, 2007, 195(4):298-306.

[34] Leary, MarkR. Understanding social anxiety[M]. Sage Publications, 1983.

[35] JI Xuan, YU Ting. Relationship between College Students ' Social Ability and Online Social Behavior[J].New Silk Road Horizon,2021(01):107$110+112$.

[36] WANG Juan, FAN Jia-lu, PAN He-ping. The Relationship between Social Support and College Students ' Social Anxiety[J].Chinese Journal of Disease Control \& Prevention,2009,13(03):293295.

[37] Arnett J J. Emerging Adulthood: What Is It, and What Is It Good For?[J]. Child Development Perspectives, 2010, 1.

[38] Palapattu A G, Kingery J N , Ginsburg G S . Gender Role Orientation and Anxiety Symptoms Among African American Adolescents[J]. Journal of Abnormal Child Psychology, 2006, 34(3):441449. 